\title{
Mapping Prostate Cancer Lesions Before and After Unsuccessful Salvage Lymph Node Dissection Using Repeat PSMA PET
}

\author{
Andrea Farolfi ${ }^{1,2}$, Harun Ilhan $^{3}$, Andrei Gafita ${ }^{4}$, Jeremie Calais ${ }^{5}$, Francesco Barbato ${ }^{2}$, Manuel Weber ${ }^{2}$, \\ Ali Afshar-Oromieh ${ }^{6,7}$, Fabian Spohn ${ }^{6}$, Axel Wetter ${ }^{8}$, Christoph Rischpler $^{2}$, Boris Hadaschik ${ }^{9}$, Davide Pianori ${ }^{10}$, \\ Stefano Fanti ${ }^{1}$, Uwe Haberkorn ${ }^{6,11}$, Matthias Eiber ${ }^{4}$, Ken Herrmann*2, and Wolfgang Peter Fendler*2,5 \\ ${ }^{1}$ Nuclear Medicine Unit, University of Bologna, S. Orsola Hospital, Bologna, Italy; ${ }^{2}$ Department of Nuclear Medicine, University of \\ Duisburg-Essen and German Cancer Consortium (DKTK), University Hospital Essen, Essen, Germany; ${ }^{3}$ Department of Nuclear \\ Medicine, University Hospital, Ludwig-Maximilians-Universität, Munich, Germany; ${ }^{4}$ Department of Nuclear Medicine, Klinikum \\ rechts der Isar, Technical University Munich, Munich, Germany; ${ }^{5}$ Ahmanson Translational Theranostics Division, Department of \\ Molecular and Medical Pharmacology, UCLA, Los Angeles, California; ${ }^{6}$ Department of Nuclear Medicine, Heidelberg University \\ Hospital, Heidelberg, Germany; ${ }^{7}$ Department of Nuclear Medicine, Bern University Hospital, University of Bern, Bern, Switzerland; \\ ${ }^{8}$ Department of Diagnostic and Interventional Radiology and Neuroradiology, University Hospital Essen, Essen, Germany; \\ ${ }^{9}$ Department of Urology, University Hospital Essen, Essen, Germany; ${ }^{10}$ Department of Biomedical and Neuromotor Sciences, \\ University of Bologna, Bologna, Italy; and ${ }^{11}$ Clinical Cooperation Unit Nuclear Medicine, German Cancer Research Center, \\ Heidelberg, Germany
}

The aim of this study was to analyze patterns of persistent versus recurrent or new PET lesions in a selected patient cohort with prostate-specific antigen (PSA) persistence after salvage lymph node dissection (SLND) and pre-procedure and post-procedure prostatespecific membrane antigen (PSMA) ligand PET. Methods: Sixteen patients were included in this multicenter study. The inclusion criteria were PSMA PET performed for biochemical recurrence before SLND (pre-SLND PET) and repeat PSMA PET performed for a persistently elevated PSA level ( $\geq 0.1 \mathrm{ng} / \mathrm{mL}$ ) at least $6 \mathrm{wk}$ after SLND (post-SLND $P E T$ ). Image analysis was performed by 3 independent nuclear medicine physicians applying the molecular imaging TNM system PROMISE. Lesions were confirmed by histopathology, presence on correlative CT/MRI/bone scanning, or PSA response after focal therapy. Results: Post-SLND PET identified prostate cancer lesions in $88 \%(14 / 16)$ of patients with PSA persistence after SLND. Median PSA was $1.2 \mathrm{ng} / \mathrm{mL}$ (interquartile range, $0.6-2.8 \mathrm{ng} / \mathrm{mL}$ ). Disease was confined to the pelvis in $56 \%$ of patients $(9 / 16)$, and most of these men had common iliac $(6 / 16,38 \%)$ and internal iliac lymph node metastases $(6 / 16,38 \%)$. Extrapelvic disease was detected in $31 \%$ of patients $(5 / 16)$. In pre- and post-SLND PET comparison, 10 of 16 had at least one lesion already detected at baseline (63\% PET persistence), 4 of 16 had new lesions only (25\% PET recurrence), and 2 had no disease on post-SLND PET. All validated regions (11 regions in 9 patients) were true-positive. Nine of 14 (64\%) patients underwent repeat local therapies after SLND (7/14 radiotherapy, 2/14 surgery). Conclusion: SLND of pelvic nodal metastases was often not complete according to PSMA PET. About two thirds of patients had PET-positive nodal disease after SLND already seen on pre-SLND PSMA PET. Notably, about one quarter of patients had new lesions, not detected by presurgical PSMA PET.

Received Aug. 25, 2019; revision accepted Nov. 18, 2019.

For correspondence or reprints contact: Wolfgang Peter Fendler, Department of Nuclear Medicine, University Hospital Essen, Hufelandstraße 55, 45147 Essen, Germany.

E-mail: wolfgang.fendler@uk-essen.de

${ }^{*}$ Contributed equally to this work.

Published online Dec. 5, 2019.

COPYRIGHT @ 2020 by the Society of Nuclear Medicine and Molecular Imaging.
Key Words: PET; prostate cancer; PSA persistence; PSMA; salvage lymph node dissection

J Nucl Med 2020; 61:1037-1042

DOI: 10.2967/jnumed.119.235374

I n cases of biochemical recurrence after radical prostatectomy, it is important to determine whether the recurrence has developed at local or distant sites. Therefore, current guidelines recommend prostate-specific membrane antigen (PSMA) ligand PET imaging if the outcome will affect treatment planning (1). Promising efficacy for metastasis-directed therapy such as salvage lymph node dissection (SLND) or salvage radiotherapy has been found in several studies $(2,3)$, but evidence of the long-term oncologic impact is missing. In patients with longer prostate-specific antigen (PSA) doubling times, lower radical-prostatectomy-specimen pathologic International Society of Urologic Pathologists grades, and disease recurrence confined to lymph nodes, metastasis-directed therapy aims at decreasing the risk of distant progression, allowing a later onset of androgen deprivation therapy, and potentially improving cancer-specific survival. PSMA PET is able to localize biochemically recurrent prostate cancer (PCa) with unprecedented accuracy even at low PSA levels (4-7). Resection of suggestive nodes is feasible; however, more than half of patients demonstrate persistently elevated PSA after SLND (8). Accurate staging is helpful to localize the source of PSA for subsequent management decisions, including surveillance, repeat localized salvage therapy, or systemic therapy. Disease locations individually guide local retreatment to balance efficacy and potential toxicity (8). Conventional imaging does not localize relevant lesions in this clinical setting, especially at low PSA levels. PSMA PET is a promising staging tool sensitive enough to uncover disease locations before and after SLND. We hypothesize that PSMA PET localizes residual and new PCa lesions in patients with PSA persistence after SLND 


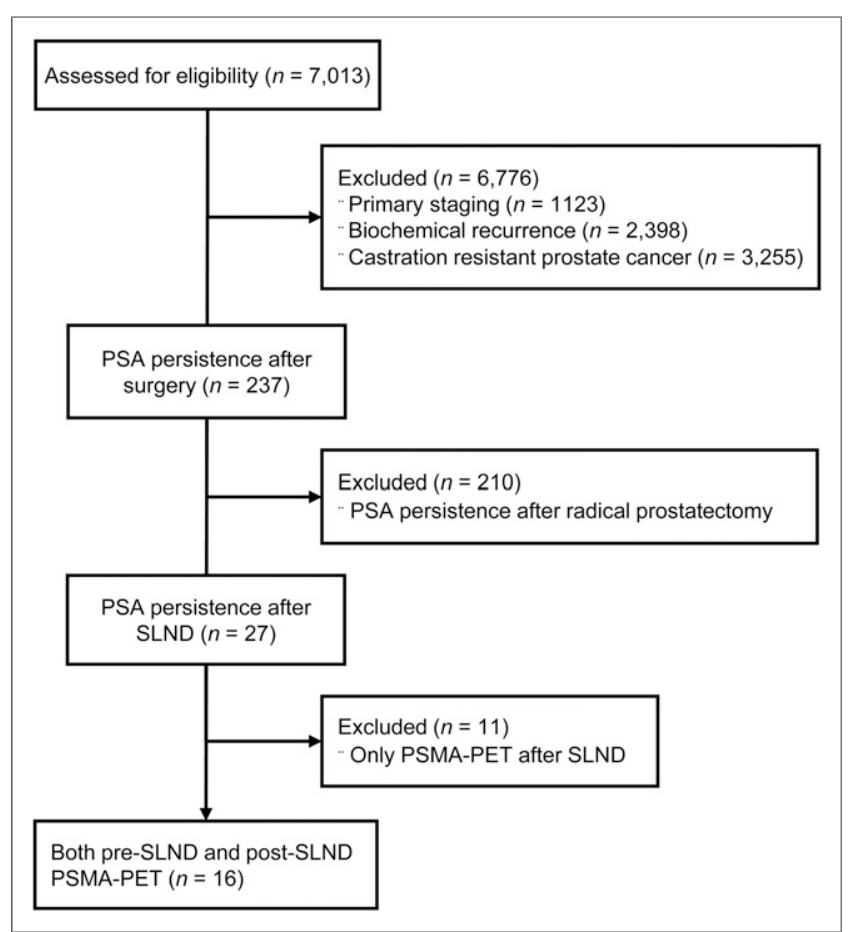

FIGURE 1. Consort diagram for patient selection.

with high accuracy. We therefore selected patients with preprocedure and postprocedure PSMA PET to better understand disease patterns of unsuccessful salvage lymph node resection.

\section{MATERIALS AND METHODS}

\section{Patient Population}

Between June 2013 and April 2018, datasets from 7,013 patients with PSMA PET for PCa were retrospectively reviewed at 6 high-volume nuclear medicine centers, and 16 patients with PSA persistence after SLND were enrolled (Fig. 1). The inclusion criteria were PSMA PET performed for biochemical recurrence of PCa before SLND (pre-SLND PET) and repeat PSMA PET performed for a persistently elevated PSA level $(\geq 0.1$ $\mathrm{ng} / \mathrm{mL}$ ) at least $6 \mathrm{wk}$ after SLND (post-SLND PET). Anonymized data were centrally collected at the Department of Nuclear Medicine of the University Duisburg-Essen, Germany. This retrospective analysis was approved by the local ethics committee (reference number 18-8136-BO), which waived the requirement to obtain informed consent for inclusion.

\section{Image Acquisition}

${ }^{68}$ Ga-PSMA-11 (Glu-NH-CO-NH-Lys-(Ahx)-[ $\left[{ }^{68} \mathrm{Ga}(\mathrm{HBED}-\mathrm{CC})\right]$ ) was used as the PSMA ligand. The median injected activity was $154 \mathrm{MBq}$ (interquartile range [IQR], 100-172 MBq). The median uptake time was $64 \mathrm{~min}$ (IQR, 56-77). Intravenous contrast was given before 26 of the $32(81 \%)$ PET/CT scans. PET imaging protocols were in accordance with the joint procedure guideline of the Society of Nuclear Medicine and Molecular Imaging and the European Association of Nuclear Medicine for PSMA PET imaging (9).

\section{Image Interpretation}

Anonymized imaging datasets were evaluated independently by 3 experienced nuclear medicine physicians with at least $2 \mathrm{y}$ of experience in PSMA PET imaging, who followed recent recommendations $(9,10)$ and were unaware of the clinical history. Training of the readers was based on 30 PSMA PET cases ranging from unremarkable to extensive disease and including typical pitfalls (11). On the basis of the molecular imaging TNM system PROMISE, the following regions and subregions were systematically rated as positive versus negative for PCa $(12,13)$ : prostate bed, pelvic nodes (internal iliac, obturator, external iliac, mesorectal, presacral, common iliac), extrapelvic nodes (retroperitoneal, mesenteric, inguinal, above diaphragm), bone, and visceral organs. In cases of discordance, consensus (PET-positive vs. -negative) was determined by a 2-versus-1 majority vote among the 3 readers.

\section{Lesion Validation and Management}

Local investigators reviewed patient files for correlative and followup information acquired during routine clinical practice. Post-SLND PET-positive findings were confirmed by histopathology, the presence of lesions on correlative CT/MRI/bone scanning, or a PSA response of more than $50 \%$ after focal therapy acquired during clinical routine. PETpositive findings were validated as true- or false-positive on a region basis.

\section{Statistical Analysis}

For continuous data, median and IQR were reported, whereas categoric variables were described using frequencies. PSMA PET detection rate for the localization of residual PCa after SLND was determined on a patient basis stratified by PSA at the time of PET and PSA nadir after SLND. The association between the PSMA PET results and the presence of local or distant lesions and PSA at the time of PET, PSA nadir, PSA doubling time, and PSA velocity was evaluated with nonparametric Mann-Whitney $U$ tests in view of their asymmetric distribution. Furthermore, individual patient data are given. Overall agreement among 3 readers was evaluated using the Fleiss $\kappa$-coefficient. All analyses were performed using the Stata software package, version 15 (StataCorp LLC).

\section{RESULTS}

Patient characteristics are given in Table 1. The median PSA nadir after SLND was $0.4 \mathrm{ng} / \mathrm{mL}$ (IQR, 0.3-2.5 ng/mL), and the median PSA value at the time of PET after SLND was $1.2 \mathrm{ng} / \mathrm{mL}$ (IQR, 0.6$2.8 \mathrm{ng} / \mathrm{mL}$ ). Individual patient characteristics and PET findings are listed in Table 2. Regarding PSA persistence, disease was localized after SLND in 14 of 16 patients, resulting in an overall detection rate of $88 \%$. PSA at the time of PET, PSA nadir, PSA doubling time, and

TABLE 1

Patient Characteristics $(n=16)$

\begin{tabular}{lc}
\hline \multicolumn{1}{c}{ Characteristic } & Data \\
\hline Age $(\mathrm{y})$ & $66(59-75)$ \\
\hline PSA initial $(\mathrm{ng} / \mathrm{mL})$ & $6.6(5.9-88.1)$ \\
PSA nadir after SLND $(\mathrm{ng} / \mathrm{mL})$ & $0.4(0.3-2.5)$ \\
PSA at time of PET $(\mathrm{ng} / \mathrm{mL})$ & $1.2(0.6-2.8)$ \\
PSA doubling time $(\mathrm{mo})$ & $1.8(0-7.2)$ \\
PSA velocity $(\mathrm{ng} / \mathrm{mL} / \mathrm{y})$ & $0.7(0-2.2)$ \\
Interval from pre-SLND PET to & $1.0(1-2)$ \\
SLND (mo) & $3.5(2-6)$ \\
Interval from SLND to post-SLND & \\
PET (mo) & $12 / 16(75 \%)$ \\
$\geq$ T3a & $6 / 13(46 \%)$ \\
N1 & $11 / 16(69 \%)$ \\
\hline ISUP grade group $\geq 4$ &
\end{tabular}

ISUP $=$ International Society of Urologic Pathologists.

Qualitative data are expressed as frequency followed by percentages in parentheses; continuous data are expressed as median followed by IQR in parentheses. 


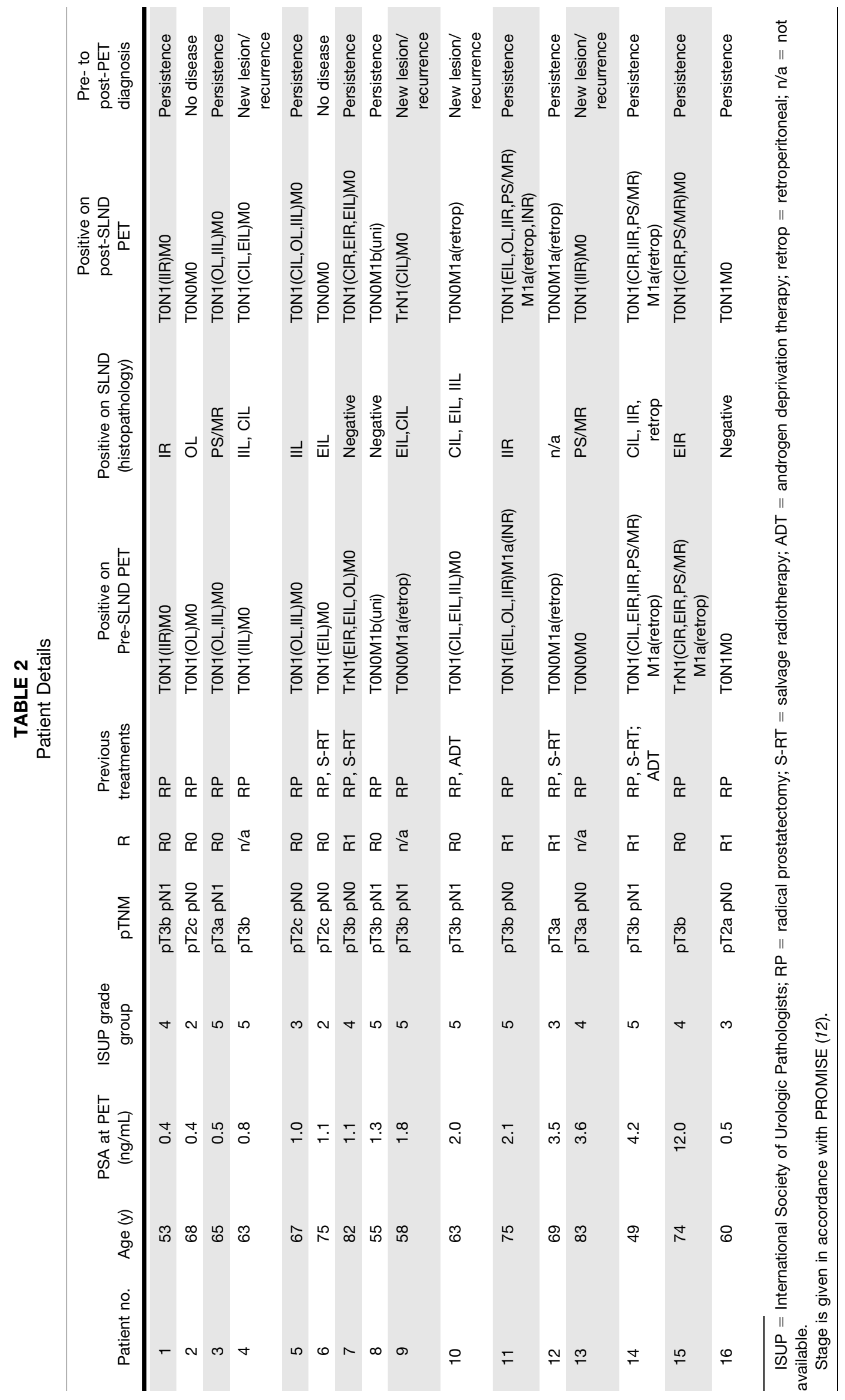




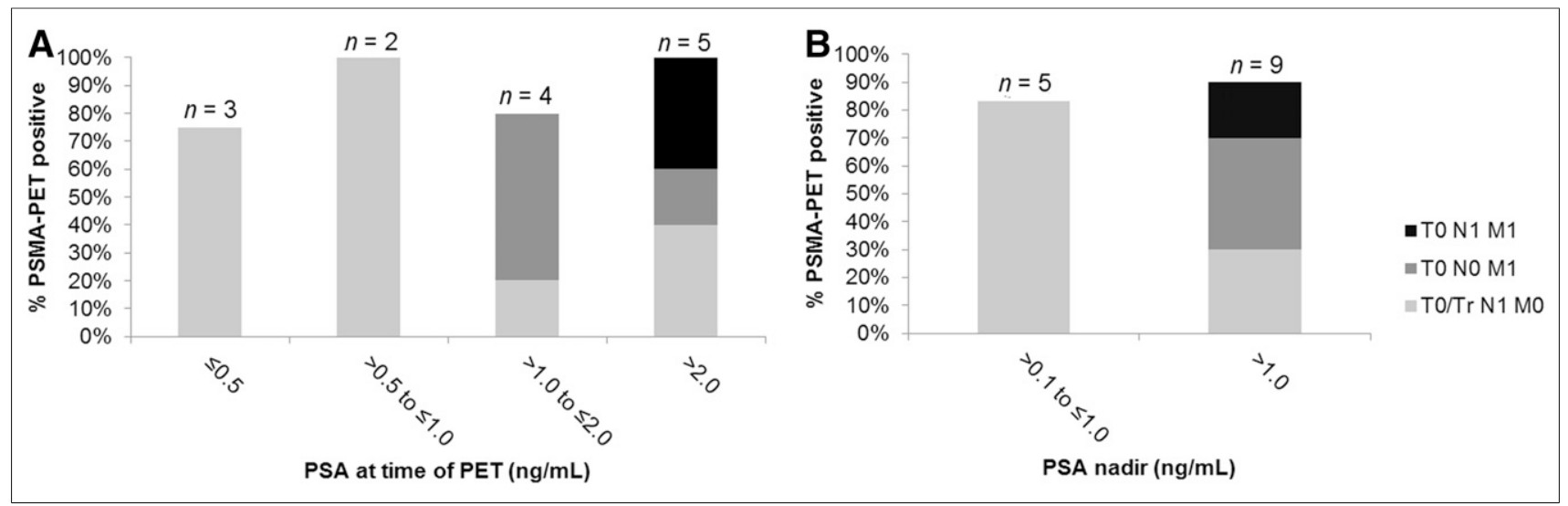

FIGURE 2. PSMA PET detection rate on patient basis stratified by PSA at time of PET (A) and PSA nadir after SLND (B).

PSA velocity were not significantly associated with the post-SLND PET result (positive or negative) (Fig. 2). In the patient-based analysis, disease confined to the pelvis was detected in $56 \%$ of patients $(9 / 16)$,

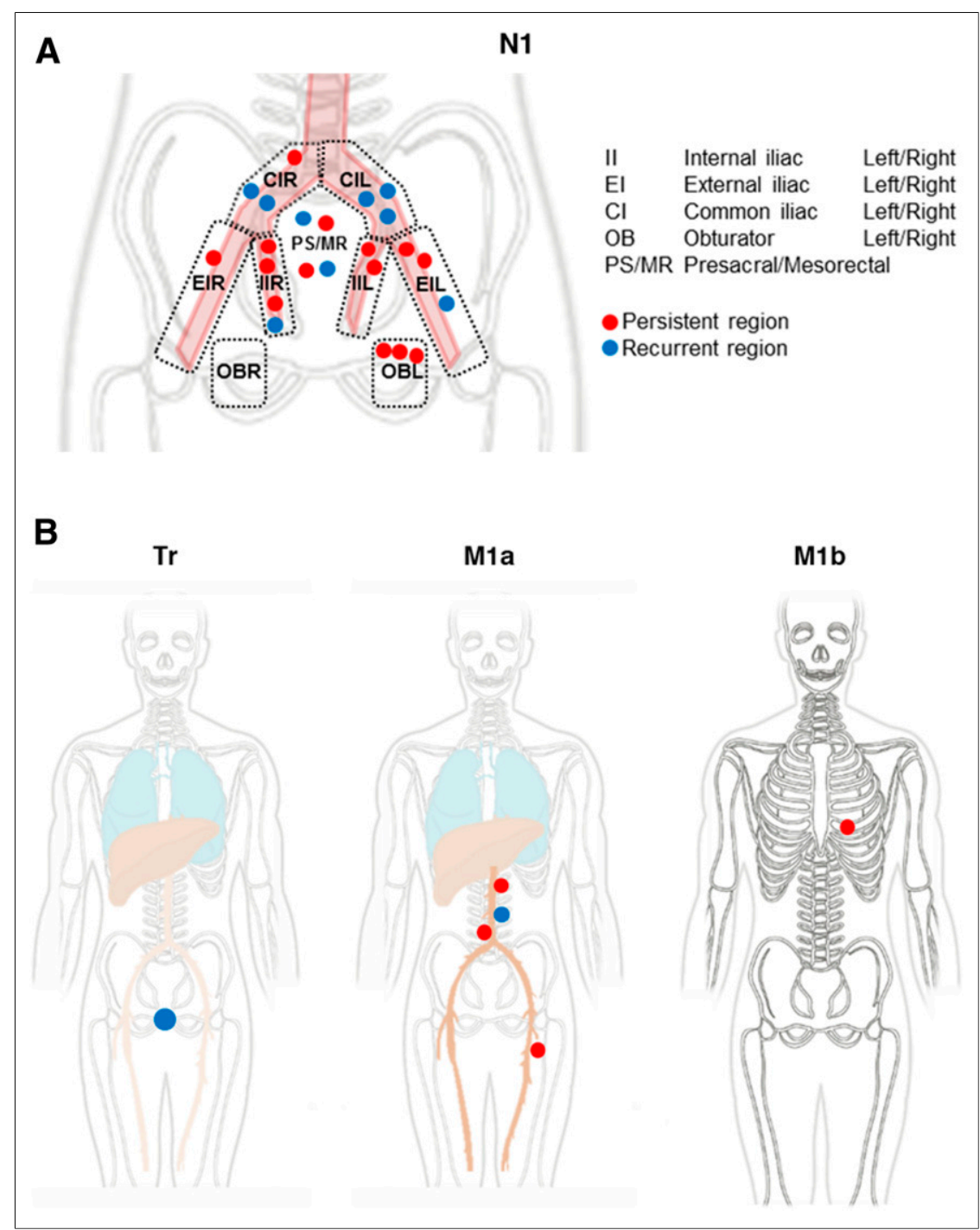

FIGURE 3. PSMA PET findings in pre- and post-SLND PET comparison ( $n=16$ patients) shown separately for pelvic lymph nodes (N1) (A) and for prostate bed (Tr), distant nodes (M1a), and bone metastases (M1b) (B). with predominant pelvic nodal disease $(9 / 16,56 \%)$ and 1 local recurrence $(1 / 16,6 \%)$. The most frequently affected pelvic nodal regions were common iliac $(6 / 16,38 \%)$ and internal iliac $(6 / 16,38 \%)$.

Distant disease was detected in $31 \%$ of patients (5/16). Extrapelvic lymph nodes were detected in 4 of 16 patients $(25 \%)$, whereas bone lesions were detected in 1 of $16(6 \%)$. There was a statistically significant association between a higher PSA $(P=0.047)$ or a shorter PSA doubling time $(P=0.018)$ at the time of PET and the presence of distant lesions. A longer PSA doubling time was significantly associated with the presence of local lesions only $(P=0.02)$. PET-positive pelvic nodes presented with intense PSMA uptake (median $\mathrm{SUV}_{\text {max }}$, 15; IQR, 9-21); however, most were not enlarged according to CT criteria (median short diameter, $0.8 \mathrm{~cm}$; IQR, 0.5-0.9).

\section{Presurgical and Postsurgical PET Comparison}

PSMA PET was performed before SLND with a median time of 1 mo (IQR, 1-2 mo). The median time between SLND and PET after SLND was 4 mo (IQR, 2-6 mo). Findings on PSMA PET before SLND are listed in Supplemental Table 1 (supplemental materials are available at http://jnm.snmjournals. org). All lesions noted on pre-SLND PET were reported for surgery planning. The median number of lymph nodes removed at SLND was 17 (IQR, 10-20). Overall, pre-SLND PET detected 24 pelvic lymph node metastases, whereas SLND resulted in 88 histopathology-positive nodes (226 nodes were removed in total).

After SLND, all men had PSA persistence (selection criteria for the current analysis). PET after SLND in these 16 patients demonstrated the following: 10 $(63 \%)$ had at least 1 lesion already detected before SLND (PET persistence or mixed), whereas $4(25 \%)$ had new lesions after SLND only (PET recurrence) (Fig. 3). The 
most frequently affected nodal regions with PET persistence were internal iliac (5/10 N1 regions), obturator (3/10 N1 regions), and external iliac ( $3 / 10 \mathrm{~N} 1$ regions). Lesions recurred most often in the common iliac (5/10) region. Two of $16(13 \%)$ patients had no disease on PET after SLND.

Patient 8 had mild focal uptake in the fifth left rib $\left(\mathrm{SUV}_{\max }, 3\right)$ before SLND. The PET findings were read as equivocal in the absence of sclerosis on CT. PET after SLND demonstrated intense focal uptake highly suggesting the presence of a unifocal bone metastasis $\left(\mathrm{SUV}_{\max }, 14\right)$. Patient examples of persistent and recurrent metastases are given in Supplemental Figures 1 and 2.

\section{Interreader Agreement, Lesion Validation, and Management}

The overall interpretation among the 3 readers had fair agreement for the local prostate bed (Fleiss $\kappa=0.33$ ) and substantial to almost-perfect agreement for pelvic nodes $(\kappa=0.69)$ and distant metastases $(\kappa=0.68)$.

Lesion validation was available for 9 of 16 patients $(56 \%)$, and 11 of 11 validated regions were true-positive: 5 with histology, 4 with imaging follow-up, and 2 with a PSA response of more than $50 \%$ after salvage radiotherapy (Table 3 ).

Management after PSMA PET was recorded for 14 of 16 patients (88\%). Nine of $14(64 \%)$ patients had repeat local therapies: patients 1 , $3,10,11,13,14$, and 16 received salvage radiotherapy with or without androgen deprivation therapy; patient 5 had repeat SLND. Patient 9 underwent salvage surgery confirming local PCa relapse with bladder invasion as well as a nodal metastasis. Patients 7 and 15 were started on androgen deprivation therapy. Patients 2,6 , and 8 had surveillance.

\section{DISCUSSION}

Patients undergoing SLND often experience PSA persistence or early relapse (8). As management of SLND failure is beyond guideline recommendations and often individually tailored, accurate staging of persistent disease is of high importance for subsequent management decisions. Treatment options include surveillance, systemic therapy, or repeat salvage attempts for localized disease.

Previously, PSMA PET demonstrated superior accuracy for the localization of recurrent PCa at low PSA values $(4,14)$. Furthermore, PSMA PET localized persistent PCa after radical prostatectomy (15). In an SLND scenario, staging of patients is crucial before proceeding with surgery. However, disease location and etiology of PSA failure have not been analyzed systematically yet. PSMA PET provides the high accuracy needed to map persistent or new lesions after unsuccessful SLND, even for subcentimeter lymph node metastases.

In this study, repeat PSMA PET was assessed in 16 patients to characterize patterns of persistent versus recurrent disease locations for unsuccessful SLND, defined as PSA persistence (PSA $\geq$ $0.1 \mathrm{ng} / \mathrm{mL}$ ) after surgery.

To our knowledge, this was the first study systematically comparing PSMA PET before and after SLND for PSA persistence. In our highly selected cohort, repeat PSMA PET was able to localize disease in nearly all patients with biochemical failure after PETinformed salvage surgery. By comparing both pre-SLND and postSLND PET, we demonstrated that lesions were already noted on PET in about two thirds of patients before salvage surgery (persistence). PET persistence was most often noted for the internal iliac and obturator regions with more difficult surgical access. The median size of PET-positive pelvic lymph nodes was $0.8 \mathrm{~cm}$ and thus below the CT and MRI size thresholds for metastatic disease $(16,17)$. Pre-SLND PET findings were known to the surgeon, and most of the PET-positive regions were surgically explored during SLND, indicating the need for improved PET guidance for the standard resection templates.

As demonstrated previously, PSMA PET outside the prostate bed was highly reproducible and predictive of PCa location $(4,15)$. However, PSMA PET before and after salvage surgery underestimated the total number of diseased nodes before SLND (17). Also, in about one quarter of the patients, the PSMA PET after SLND showed lesions that were not visible before SLND, rated as PET recurrence. PSA persistence indicates that lesions may have been present before surgery but were not picked up because of submaximal sensitivity of PSMA PET. Deficiencies for the detection of small metastases have been characterized previously (17). Despite underestimation on a single-lesion level, identification of diseased regions informs targeted therapy, including radiotherapy and repeat surgery, especially in a PSMA radioguided setting (18). Focal therapy guided by PSMA PET may lead to effective reduction of serum PSA levels (4).

In our study, about two thirds of patients underwent repeat local therapy after SLND failure. The addition of adjuvant radiotherapy might be able to improve local recurrence-free survival (19). In this setting, patients might benefit from accurate PSMA PET staging (20,21); however, prospective randomized evidence is needed to assess oncologic outcomes.

TABLE 3

Lesion Validation

\begin{tabular}{clll}
\hline Patient & Validated region & Type of validation & Validation result \\
\hline 1 & $\mathrm{~N} 1$ & PSA response after S-RT & True-positive \\
\hline 5 & $\mathrm{~N} 1$ & Surgery & True-positive \\
\hline 8 & $\mathrm{M} 1 \mathrm{~b}$ & Imaging follow-up & True-positive \\
\hline 9 & $\mathrm{Tr}, \mathrm{N} 1$ & Surgery & True-positive \\
11 & $\mathrm{~N} 1, \mathrm{M} 1 \mathrm{a}$ & Surgery, imaging follow-up & True-positive \\
\hline 13 & $\mathrm{~N} 1$ & PSA response after S-RT & True-positive \\
\hline 14 & $\mathrm{~N} 1, \mathrm{M} 1 \mathrm{a}$ & Surgery, imaging follow-up & True-positive \\
15 & $\mathrm{~N} 1$ & Surgery & True-positive \\
16 & $\mathrm{~N} 1$ & Imaging follow-up & True-positive \\
\hline
\end{tabular}

$\mathrm{S}-\mathrm{RT}=$ salvage radiotherapy. 
Furthermore, patients demonstrated a short PSA doubling time, highlighting that patient selection for salvage interventions should not only be based on imaging but also consider PSA kinetics and pathologic grades.

Limitations of the present study are its retrospective design and the small cohort size of a highly selected patient group. Bias is introduced by selection of intraindividual pre- and post-SLND PSMA PET pairs, which are typically not available in the standard SLND setting. Patient characteristics and increased imaging indicate high risk, which may have led to an overestimation of detection rates. Findings may not be applicable to a general cohort of patients with SLND and PSA persistence. Moreover, surgical technique and eligibility criteria for SLND were not standardized among the enrolling centers. Findings might thus be not representative of the clinical SLND scenario.

\section{CONCLUSION}

Comparison of pre- and postprocedure PSMA PET revealed disease patterns in patients with biochemical persistence after SLND: PSMA PET identified locations of persistent disease in about two thirds of patients. Post-SLND PET further detected new or potentially growing metastases. About two thirds of patients underwent repeat local therapies after unsuccessful SLND, indicating the potential value of accurate PSMA PET staging for PSA persistence.

\section{DISCLOSURE}

Wolfgang Fendler is a consultant for Ipsen, Endocyte, and BTG, and he received personal fees from RadioMedix outside the submitted work. He also received financial support from the German Research Foundation (Deutsche Forschungsgemeinschaft, DFG, grant FE1573/ 3-1/659216), Mercator Research Center Ruhr (MERCUR, An-20190001), IFORES (D/107-81260, D/107-30240), Doktor Robert PflegerStiftung, and Wiedenfeld-Stiftung/Stiftung Krebsforschung Duisburg. Ken Herrmann received personal fees from Bayer, Sofie Biosciences, SIRTEX, ABX, Adacap, Curium, and Endocyte; grants and personal fees from BTG; personal fees from IPSEN; personal fees and nonfinancial support from Siemens Healthineers; and nonfinancial support from GE Healthcare, outside the submitted work. Boris Hadaschik received grants from German Cancer Aid, German Research Foundation, and Profound Medical; grants, personal fees, and nonfinancial support from Janssen; personal fees and nonfinancial support from Astellas, Bayer, BMS, Lightpoint Medical, Astra Zeneca, and Sanofi; and grants and personal fees from Uromed, all outside the submitted work. No other potential conflict of interest relevant to this article was reported.

\section{KEY POINTS}

QUESTION: Where is persistent or recurrent $\mathrm{PCa}$ located in patients with unsuccessful SLND?

PERTINENT FINDINGS: In this retrospective multicenter cohort study assessing pre- and postprocedure PSMA PET to localize $\mathrm{PCa}$ in patients with PSA persistence after SLND, PSMA PET/CT localized residual disease in nearly all patients, most often in the internal iliac and obturator regions. About one quarter of patients had new lesions, not detected by presurgical PSMA PET.

IMPLICATIONS FOR PATIENT CARE: PSMA PET localized PCa both before and after unsuccessful SLND. Lesion persistence was most often noted for regions with difficult surgical access.

\section{REFERENCES}

1. Mottet N, van den Bergh RCN, Briers E, et al. Prostate cancer. European Association of Urology website.. https://uroweb.org/guideline/prostate-cancer/\#11. Published 2019. Accessed May 14, 2020.

2. Ost P, Reynders D, Decaestecker K, et al. Surveillance or metastasis-directed therapy for oligometastatic prostate cancer recurrence: a prospective, randomized, multicenter phase II trial. J Clin Oncol. 2018;36:446-453.

3. De Bleser E, Jereczek-Fossa BA, Pasquier D, et al. Metastasis-directed therapy in treating nodal oligorecurrent prostate cancer: a multi-institutional analysis comparing the outcome and toxicity of stereotactic body radiotherapy and elective nodal radiotherapy. Eur Urol. 2019;76:732-739.

4. Fendler WP, Calais J, Eiber M, et al. Assessment of ${ }^{68}$ Ga-PSMA-11 PET accuracy in localizing recurrent prostate cancer: a prospective single-arm clinical trial. JAMA Oncol. 2019;5:856-863.

5. Eiber M, Maurer T, Souvatzoglou M, et al. Evaluation of hybrid ${ }^{68} \mathrm{Ga}-\mathrm{PSMA}$ ligand PET/CT in 248 patients with biochemical recurrence after radical prostatectomy. J Nucl Med. 2015;56:668-674.

6. Afshar-Oromieh A, Avtzi E, Giesel FL, et al. The diagnostic value of PET/ CT imaging with the ${ }^{68} \mathrm{Ga}$-labelled PSMA ligand HBED-CC in the diagnosis of recurrent prostate cancer. Eur J Nucl Med Mol Imaging. 2015;42:197209.

7. Afshar-Oromieh A, Holland-Letz T, Giesel FL, et al. Diagnostic performance of ${ }^{68} \mathrm{Ga}-\mathrm{PSMA}-11$ (HBED-CC) PET/CT in patients with recurrent prostate cancer: evaluation in 1007 patients. Eur J Nucl Med Mol Imaging. 2017;44: 1258-1268.

8. Ploussard G, Gandaglia G, Borgmann H, et al. Salvage lymph node dissection for nodal recurrent prostate cancer: a systematic review. Eur Urol. 2019;76: 493-504.

9. Fendler WP, Eiber M, Beheshti M, et al. ${ }^{68} \mathrm{Ga}$-PSMA PET/CT: joint EANM and SNMMI procedure guideline for prostate cancer imaging: version 1.0. Eur J Nucl Med Mol Imaging. 2017;44:1014-1024.

10. Rauscher I, Maurer T, Fendler WP, Sommer WH, Schwaiger M, Eiber M. ${ }^{68} \mathrm{Ga}-$ PSMA ligand PET/CT in patients with prostate cancer: how we review and report. Cancer Imaging. 2016;16:14.

11. Fendler WP, Calais J, Allen-Auerbach M, et al. ${ }^{68}$ Ga-PSMA-11 PET/CT interobserver agreement for prostate cancer assessments: an international multicenter prospective study. J Nucl Med. 2017;58:1617-1623.

12. Eiber M, Herrmann K, Calais J, et al. Prostate Cancer Molecular Imaging Standardized Evaluation (PROMISE): proposed miTNM classification for the interpretation of PSMA-ligand PET/CT. J Nucl Med. 2018;59:469-478.

13. Taylor A, Rockall AG, Powell MEB. An atlas of the pelvic lymph node regions to aid radiotherapy target volume definition. Clin Oncol (R Coll Radiol). 2007;19: 542-550.

14. Calais J, Ceci F, Eiber M, et al. ${ }^{18} \mathrm{~F}$-fluciclovine PET-CT and ${ }^{68} \mathrm{Ga}$-PSMA-11 PET-CT in patients with early biochemical recurrence after prostatectomy: a prospective, single-centre, single-arm, comparative imaging trial. Lancet Oncol. 2019;20:1286-1294.

15. Farolfi A, Gafita A, Calais J, et al. ${ }^{68} \mathrm{Ga}$-PSMA-11 positron emission tomography detects residual prostate cancer after prostatectomy in a multicenter retrospective study. J Urol. 2019;202:1174-1181.

16. Mandel P, Tilki D, Chun FK, et al. Accuracy of ${ }^{68}$ Ga-prostate-specific membrane antigen positron emission tomography for the detection of lymph node metastases before salvage lymphadenectomy. Eur Urol Focus. 2020;6:7173.

17. Jilg CA, Drendel V, Rischke HC, et al. Diagnostic accuracy of Ga-68-HBEDCC-PSMA-ligand-PET/CT before salvage lymph node dissection for recurrent prostate cancer. Theranostics. 2017;7:1770-1780.

18. Horn T, Krönke M, Rauscher I, et al. Single lesion on prostate-specific membrane antigen-ligand positron emission tomography and low prostate-specific antigen are prognostic factors for a favorable biochemical response to prostate-specific membrane antigen-targeted radioguided surgery in recurrent prostate cancer. Eur Urol. 2019;76:517-523.

19. Rischke HC, Schultze-Seemann W, Wieser G, et al. Adjuvant radiotherapy after salvage lymph node dissection because of nodal relapse of prostate cancer versus salvage lymph node dissection only. Strahlenther Onkol. 2015;191: 310-320.

20. Maurer T, Robu S, Schottelius M, et al. ${ }^{99 \mathrm{~m}}$ Technetium-based prostate-specific membrane antigen-radioguided surgery in recurrent prostate cancer. Eur Urol. 2019;75:659-666.

21. Rauscher I, Düwel C, Wirtz M, et al. Value of ${ }^{111}$ In-prostate-specific membrane antigen (PSMA)-radioguided surgery for salvage lymphadenectomy in recurrent prostate cancer: correlation with histopathology and clinical followup. BJU Int. 2017;120:40-47. 